\title{
A Galton-Watson Estimate for Dyson Series
}

\author{
Hans Maassen and Dmitri Botvich
}

We dedicate this paper to John T. Lewis, who was a teacher and source of inspiration to both of us.

\begin{abstract}
We consider the question of convergence of particular series of integrals, which are labeled by rooted trees. Necessary and sufficient criteria for convergence are obtained, together with an explicit expression for the sum. The technique used is strongly reminiscent of the generating function approach of Galton and Watson to branching processes. The interest in these series derives from the Dyson series expansion for the perturbation of a free quantum dynamics by a local potential: the convergence of the series implies that the perturbed dynamics exists and is unitarily equivalent with the free one.
\end{abstract}

\section{Introduction}

The purpose of this paper is to provide a technical result that can be used in the perturbation theory of infinite quantum systems. It provides a summability condition, and an upper bound, for the Dyson series associated to local perturbations of well-behaved 'free' time evolutions.

It is of direct relevance in the quasi-particle description of bosonic and fermionic systems [3], the study of approach to thermal equilibrium in Caldeira-Leggett type models [17] and anharmonic chrystals [6,7], and the description of Rayleigh scattering [19] and dissipative transport [9].

The result has been available in preprint form for some time [2], and has already been applied in the derivation of Green-Kubo formulas and Onsager reciprocity relations for coupled Fermi systems [11]. Here we give a formulation and a proof with only a sketch of the context.

\subsection{Asymptotic Completeness}

The key issue in the applications is asymptotic completeness: if the scattering operator associated to the perturbation is onto, it establishes an equivalence between the free and the perturbed dynamics. The ergodic properties of the free evolution are then preserved by the perturbation. 
This scattering approach to infinite quantum systems was initiated by Robinson [18]; we give a sketch here for the $C^{*}$ situation; with some care it extends to the von Neumann algebra context, where the continuity assumptions are less restrictive.

A quantum dynamical system is a triple $\left(\mathcal{A}, \omega, \alpha_{t}\right)$, where $\mathcal{A}$ is a $C^{*}$-algebra, $\omega$ is a state on $\mathcal{A}$, and $\left(\alpha_{t}\right)_{t \in \mathbb{R}}$ a strongly continuous one-parameter group of *-automorphisms of $\mathcal{A}$. By the Gel'fand-Naimark-Segal (GNS) construction the pair $(\mathcal{A}, \omega)$ determines a Hilbert space $\mathcal{H}$, a unit vector $\xi \in \mathcal{H}$, and a representation of $\mathcal{A}$ as an algebra of bounded operators on $\mathcal{H}$ such that $\mathcal{A} \xi$ is dense in $\mathcal{H}$ and $\langle\xi, A \xi\rangle=\omega(A)$ for all $A \in \mathcal{A}$. Let us assume that the dynamical system $\left(\mathcal{A}, \omega, \alpha_{t}\right)$ is mixing, i.e. for all $A \in \mathcal{A}$ and all unit vectors $\psi \in \mathcal{H}$ we have:

$$
\lim _{t \rightarrow \infty}\left\langle\psi, \alpha_{t}(A) \psi\right\rangle=\omega(A) .
$$

The dynamics $\alpha_{t}$ determines a one-parameter group of unitary operators $\left(U_{t}\right)_{t \in \mathbb{R}}$ on $\mathcal{H}$ by the relation $U_{t} A \xi=\alpha_{t}(A) \xi$. The generator $H$ of this group, given by $U_{t}=\mathrm{e}^{i t H}$, is the Hamiltonian of the quantum dynamical system. Now let some self-adjoint element $V$ of $\mathcal{A}$ be given, and let us define new dynamics on $\mathcal{A}$ by

$$
\widetilde{\alpha}_{t}: \mathcal{A} \rightarrow \mathcal{A}: A \mapsto \mathrm{e}^{i t(H+V)} A \mathrm{e}^{-i t(H+V)} .
$$

In order to compare the two evolutions $\alpha_{t}$ and $\widetilde{\alpha}_{t}$, Robinson proposed to consider the scattering operator $\gamma$ given by the pointwise norm limit

$$
\gamma(A):=\lim _{t \rightarrow \infty} \widetilde{\alpha}_{-t} \circ \alpha_{t}(A),
$$

which exists under the fairly mild integrability condition

$$
\int_{0}^{\infty}\left\|\left[\alpha_{t}(V), A\right]\right\| \mathrm{d} t<\infty .
$$

The operator $\gamma$ intertwines the two evolutions:

$$
\gamma \circ \alpha_{t}(A)=\widetilde{\alpha}_{t} \circ \gamma(A), \quad(t \in \mathbb{R}, A \in \mathcal{A}) .
$$

Suppose further that there is another vector $\widetilde{\xi} \in \mathcal{H}$ such that the state $\widetilde{\omega}: A \mapsto$ $\langle\widetilde{\xi}, A \widetilde{\xi}\rangle$ is invariant for the perturbed evolution, and that the space of vectors $A \xi$ for which $\gamma(A)$ exists, is dense in $\mathcal{H}$. Then it is not difficult to show (cf. [16]) that the operator $\Gamma_{0}: A \xi \mapsto \gamma(A) \widetilde{\xi}$ extends to an isometry $\Gamma: \mathcal{H} \rightarrow \mathcal{H}$. In general, $\Gamma$ need not be unitary, but if it is, the scattering operator is said to be asymptotically complete, and the two evolutions $\alpha_{t}$ and $\widetilde{\alpha}_{t}$ are unitarily equivalent.

For the invertibility of $\Gamma$ it suffices that the inverse limit

$$
\widetilde{\gamma}(A):=\lim _{t \rightarrow \infty} \alpha_{-t} \circ \widetilde{\alpha}_{t}(A),
$$

exists for sufficiently many $A \in \mathcal{A}$. This is in general much harder to prove. But again there is a sufficient condition: the summability of the Dyson series over all times: for all $A \in \mathcal{A}$, 


$$
\left.\sum_{n=0}^{\infty} \int_{0 \leq t_{1} \leq \cdots \leq t_{n}} \ldots \int_{\|} \| \alpha_{-t_{n}}(V),\left[\ldots\left[\alpha_{-t_{1}}(V), A\right] \ldots\right]\right] \| \mathrm{d} t_{1} \mathrm{~d} t_{2} \ldots \mathrm{d} t_{n}<\infty .
$$

In some cases, including the applications mentioned above, this Dyson series can be bounded by a sum over rooted trees. Two examples will be treated in Sect. 3 . Due to the good combinatorial properties of trees, we thus obtain all-time integrability of the series in a definite, non-vanishing regime of perturbations $V$. This is the subject of Theorem 1 .

The result is an improvement on estimates which were used in the older cases mentioned above. It is optimal in the sense that it becomes an equality if all contributions are non-negative. We will state it in Sect. 2, and give a proof in Sect. 4.

\section{The Main Result}

Let $m_{0}, m_{1}, m_{2}, \ldots$ and $\widetilde{m}_{0}, \widetilde{m}_{1}, \widetilde{m}_{2}, \ldots$ be two sequences of non-negative numbers, and $f, \widetilde{f}$ two non-negative integrable functions on $[0, \infty)$. Assume that the numbers $\widetilde{m}_{1}, \widetilde{m}_{2}, \ldots$ are not all zero. We consider the sum of integrals

$$
\begin{aligned}
\Phi(\widetilde{m}, m, \tilde{f}, f):= & \sum_{n=0}^{\infty} \sum_{c_{1}=0}^{0} \sum_{c_{2}=0}^{1} \sum_{c_{3}=0}^{2} \cdots \sum_{c_{n}=0}^{n-1} \widetilde{m}_{d_{c}(0)}\left(\prod_{j=1}^{n} m_{d_{c}(j)}\right) \\
& \left.\times \int_{0 \leq t_{1} \leq \ldots \leq t_{n}} \ldots \int_{j=1}\left(\prod_{c_{j}}^{n} f_{c_{j}}-t_{c_{j}}\right)\right) \mathrm{d} t_{1} \mathrm{~d} t_{2} \ldots \mathrm{d} t_{n}
\end{aligned}
$$

where $t_{0}=0$ and, for $j=0,1, \ldots, n$,

$$
d_{c}(j):=\#\left\{i \in\{1,2, \ldots, n\} \mid c_{i}=j\right\}
$$

and

$$
f_{i}:= \begin{cases}\tilde{f} & \text { if } i=0 \\ f & \text { if } i \geq 1\end{cases}
$$

Let the generating functions $G, \widetilde{G}:[0, \infty) \rightarrow[0, \infty]$ be defined by

$$
G(x):=\sum_{k=0}^{\infty} \frac{m_{k}}{k !} x^{k} \quad \text { and } \quad \widetilde{G}(x):=\sum_{k=0}^{\infty} \frac{\widetilde{m}_{k}}{k !} x^{k},
$$

and let $\|f\|,\|\tilde{f}\|$ denote the integrals of $f$ and $\tilde{f}$, respectively.

Theorem 1. The sum $\Phi(\widetilde{m}, m, \widetilde{f}, f)$ in (3) converges if and only if the equation

$$
G(\|f\| y)=y
$$

allows a solution $y$ for which $\widetilde{G}(\|\widetilde{f}\| y)<\infty$. If $y$ is the least such solution, then

$$
\Phi(\widetilde{m}, m, \widetilde{f}, f)=\widetilde{G}(\|\widetilde{f}\| y) .
$$

Condition (7) is illustrated in Fig. 1. 


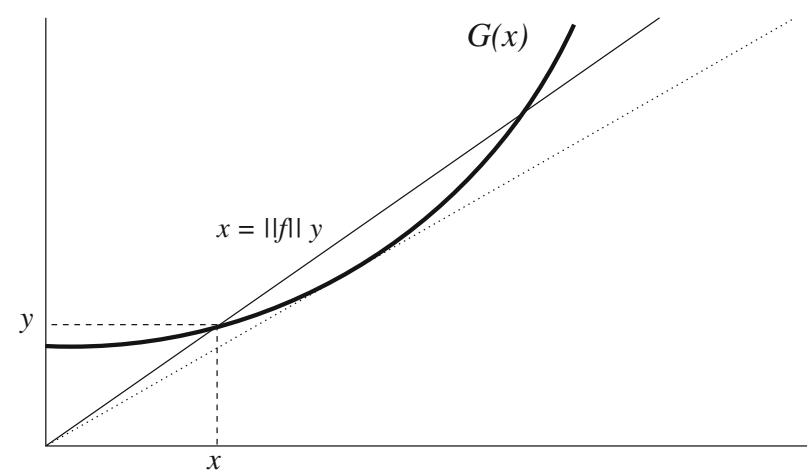

FIGURE 1. The convergence condition.

\subsection{Discussion}

At first sight, the chances for convergence of the series (3) may look slim. The number of integrals grows as $(n-1)$ !, and the integrals themselves seem to behave roughly like $\|f\|^{n}$. Hence the older estimates $[17,19]$ made a stronger requirement on $f$ than integrability, namely exponential decrease. It was clear, however, from work of Botvich et al. [1] that something better should be possible. The basic trick of the present paper is to reduce the number of terms in the sum from factorial to roughly a power law in $n$ by 'packing' many terms into a single integral. (See the 'packing lemma', Lemma 5.)

Apart from this integration aspect, there is also the summation aspect, related to the classical theory of Galton and Watson on family trees, which makes exact calculation of the sum possible. We hope to shed some light on these aspects separately in the following two corollaries to Theorem 1.

Corollary 2. Let $f:[0, \infty) \rightarrow[0, \infty)$ be Lebesgue integrable. Then for all $n \in \mathbb{N}$ :

$$
\begin{aligned}
\int_{0 \leq t_{1} \leq \cdots \leq t_{n}} & \cdots \int_{0} f\left(t_{1}\right)\left(f\left(t_{2}\right)+f\left(t_{2}-t_{1}\right)\right)\left(f\left(t_{3}\right)+f\left(t_{3}-t_{1}\right)+f\left(t_{3}-t_{2}\right)\right) \\
& \times \cdots \times\left(f\left(t_{n}\right)+f\left(t_{n}-t_{1}\right)+\cdots+f\left(t_{n}-t_{n-1}\right)\right) \mathrm{d} t_{1} \mathrm{~d} t_{2} \ldots \mathrm{d} t_{n} \\
= & \frac{(n+1)^{n-1}}{n !}\left(\int_{0}^{\infty} f(t) \mathrm{d} t\right)^{n} .
\end{aligned}
$$

We note that this expression grows roughly like $\mathrm{e}^{n}\|f\|^{n}$, and is therefore summable over $n$ for $\|f\|<\frac{1}{e}$.

Proof. Let us put $\widetilde{m}_{k}=m_{k}=1(k \in \mathbb{N}),\|f\|=\|\widetilde{f}\|=: u$. Then $\widetilde{G}(x)=G(x)=\mathrm{e}^{x}$, and Theorem 1 implies that the sum $\Phi(u):=\Phi(\widetilde{m}, m, \tilde{f}, f)$ satisfies

$$
\Phi(u)=\mathrm{e}^{u \Phi(u)} .
$$




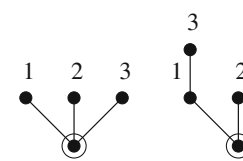

(a) (b)

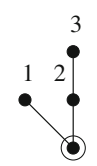

(c)

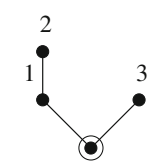

(d)

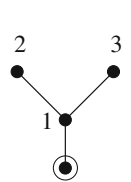

(e)

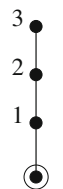

(f)

Figure 2. Rooted trees labeling the integrals.

So, putting $A(u):=u \Phi(u)$ we find

$$
A(u)=u \mathrm{e}^{A(u)} .
$$

$A$ and $\Phi$ are known as the generating functions of the combinatorial species 'rooted tree' and 'forest' in the sense of Joyal [12] (see [4]). $A$ is the inverse of the function $z \mapsto z \mathrm{e}^{-z}$, which can be found using Lagrange's inversion formula. The result for $\Phi$ is then

$$
\Phi(u)=\sum_{n=0}^{\infty} \frac{(n+1)^{n-1}}{n !} u^{n} .
$$

In particular, the $n$th term of the sum in $(3)$ is $(n+1)^{n-1}\|f\|^{n} / n$ !.

Corollary 2 shows how Theorem 1 'packs' a series of integrals over ordered $n$-tuples of times into a weighted sum over rooted trees of the $n$-fold integral over $[0, \infty)$.

Let us illustrate the combinatorial mechanisms involved in more detail, by looking at the case $n=3$ in Corollary 2. It contains six terms. For each term we make a tree diagram (Fig. 2) with four vertices, numbered 0, 1, 2, and 3, by drawing a line from vertex $i$ to vertex $j$ for each factor $f\left(t_{i}-t_{j}\right)$, and a line from $i$ to the 'root' 0 for each factor $f\left(t_{i}\right)$.

Now, integral (a) yields $\frac{1}{6}\left(\int f\right)^{3}$, since it is the integral of a symmetric function over one sixth of $[0, \infty)^{3}$ :

$$
\iint_{0 \leq t_{1} \leq t_{2} \leq t_{3}} \int_{1} f\left(t_{1}\right) f\left(t_{2}\right) f\left(t_{3}\right) \mathrm{d} t_{1} \mathrm{~d} t_{2} \mathrm{~d} t_{3}=\frac{1}{6} \int_{0}^{\infty} \int_{0}^{\infty} \int_{0}^{\infty} f\left(t_{1}\right) f\left(t_{2}\right) f\left(t_{3}\right) \mathrm{d} t_{1} \mathrm{~d} t_{2} \mathrm{~d} t_{3} .
$$

Integral (f) yields $\left(\int f\right)^{3}$ via the change of variables $u_{1}:=t_{1}, u_{2}:=t_{2}-t_{1}$, $u_{3}:=t_{3}-t_{2}$. Integral (e) can be written

$$
\int_{0}^{\infty} f\left(t_{1}\right)\left(\int_{t_{1}}^{\infty} \int_{t_{2}}^{\infty} f\left(t_{2}-t_{1}\right) f\left(t_{3}-t_{1}\right) \mathrm{d} t_{3} \mathrm{~d} t_{2}\right) \mathrm{d} t_{1}
$$

which leads to $\frac{1}{2}\left(\int f\right)^{3}$ via a change of variable $u_{2}:=t_{2}-t_{1}, u_{3}:=t_{3}-t_{1}$, and a symmetry argument similar to that for term (a). And finally, integrals (c), (b) 
and (d) come from one and the same type of tree. They can be packed together to yield $\left(\int f\right)^{3}$ as follows (Fig. 2):

$$
\begin{aligned}
& \int_{0}^{\infty} f(u) \int_{u}^{\infty} f(s-u)\left(\int_{0}^{u} f(t) \mathrm{d} t+\int_{u}^{s} f(t) \mathrm{d} t+\int_{s}^{\infty} f(t) \mathrm{d} t\right) \mathrm{d} s \mathrm{~d} u \\
& =\left(\int_{0}^{\infty} f(t) \mathrm{d} t\right)^{3} .
\end{aligned}
$$

So we see here that each type of tree diagram yields the amount $\left(\int f\right)^{3}$ divided by the number of its symmetries (or automorphisms). The number of terms reduces from six to four. Adding the four terms we find, as announced,

$$
\left(\frac{1}{6}+1+\frac{1}{2}+1\right)\left(\int f\right)^{3}=\frac{8}{3}\left(\int f\right)^{3}=\frac{(3+1)^{3-1}}{3 !}\left(\int f\right)^{3} \text {. }
$$

The second aspect of Theorem 1 is connected to the Galton-Watson theory of branching processes (e.g. [10]). In its original form this theory was concerned with the extinction of family names.

Suppose that every male in a family has probability $p_{j}$ to produce $j$ male offspring in the next generation, each of which independently produces an identically distributed number of (male) descendants in the next, etcetera. If we start with a single individual bearing a certain unique surname, what is the probability that this surname will eventually die out? (The Nineteenth Century scholars did not anticipate women's liberation developments.)

Here we do not give the textbook argument, but one which suits our purpose: Since the alternative to extinction is an infinite family tree, the probability of extinction must be the sum over all finite family trees of the probabilities of their realization. It is a small combinatorial puzzle to check that this amounts to

$$
\sum_{\text {types of rooted trees }} \frac{1}{\#(\text { symmetries tree })} \prod_{\text {vertices } v} d(v) ! p_{d(v)},
$$

where $d(v)$ stands for the number of descendants of the individual at vertex $v$.

Corollary 3. The extinction probability equals the smallest fixed point of the probability generating function

$$
G(x):=\sum_{n=0}^{\infty} p_{n} x^{n} .
$$

This is the familiar result of Galton-Watson theory.

In fact, we could even come closer to Theorem 1 by giving the first individual a different offspring distribution from his descendants. Indeed, in the quantum mechanical application the root is formed by the arbitrary observable $A$, and all other nodes represent the perturbation potential $V$. 
Proof. In Theorem 1 put

$$
\tilde{m}=m, \quad \tilde{f}=f, \quad \sum_{k=0}^{\infty} \frac{m_{k}}{k !}=1, \quad \text { and } \quad\|f\|:=\int_{0}^{\infty} f(t) \mathrm{d} t=1 .
$$

Then the theorem allows the following interpretation. In a branching process a single individual splits at time 0 into $k$ new individuals with probability $m_{k} / k$ ! . These in their turn live for independent random times, all with probability density $f$, and produce independent offspring according to the same law $m_{k} / k$ !, which again live for independent random times, distributed according to $f$, etcetera. Then the sum $\Phi(m, m, f, f)$ in (3) is the total probability measure carried by all possible finite family trees, which equals the probability that the progeny of the original individual will eventually die out.

\subsection{Earlier Results}

The case of Corollary 2 was studied by Botvich et al. [1] in the context of network theory. There the $n$th term was estimated by $(8\|f\|)^{n}$. Our result is also, in this special case, slightly better than the result of [6] which gives for the $n$th term the estimate

$$
2^{n} t^{n} \sqrt{e} c^{\frac{n \log \log n}{\log n}}, \quad(c>e) .
$$

But note that (8) and (10) are equalities, not inequalities.

In $[17,19]$ an estimate was given by requiring exponential decay of $f(t)$ as $t \rightarrow \infty$ instead of just integrability.

\section{Applications}

Now, in what kind of situations can we expect the estimate of Theorem 1 to apply to the Dyson series (2)? Let us fix self-adjoint operators $A$ and $V$ in $\mathcal{A}$.

First, it is important that the commutators

$$
\left[\alpha_{-t}(V), A\right] \text { and }\left[\alpha_{-t}(V), V\right]
$$

tend to zero as $t$ tends to infinity in an integrable way. This is an instance of a property called ' $L$-asymptotic abelianness', and expresses the fact that both $A$ and $V$ are local observables, which eventually get separated by the dynamics $\alpha_{t}$. It leads to the integrability of the functions $\widetilde{f}$ and $f$.

Second, every added commutator $\left[\alpha_{-t}(V), \cdot\right]$, acting on a repeated commutator $X=\left[\alpha_{-t_{n}}(V),\left[\ldots\left[\alpha_{-t_{1}}(V), A\right] \ldots\right]\right]$ already present, should be bounded by a sum of the form

$$
\sum_{j=1}^{n} f_{j}\left(t-t_{j}\right)\left\|X_{j}^{\prime}\right\|
$$

where $X_{j}^{\prime}$ is an expression similar to $X$, with possibly some minor alteration related to $t_{j}$, and where $f_{0}(t):=\widetilde{f}(t)$ and $f_{j}(t-s):=f(t-s)$ for $j \geq 1$ are bounds for 
$\left\|\left[\alpha_{-t}(V), A\right]\right\|$ and $\left\|\left[\alpha_{-t}(V), \alpha_{-s}(V)\right]\right\|$, respectively. This formulation is necessarily vague, every application having its own peculiarities. Instead of striving for generality, let us illustrate the transition from the Dyson series in (2) to the sum $\Phi(\widetilde{m}, m, \tilde{f}, f)$ in $(3)$ in two typical cases.

\subsection{Anharmonic Oscillator in a Bath of Oscillators}

Oscillator models are a recurrent theme in the literature on approach to equilibrium, from the ancient model of Horace Lamb [14], via the harmonic chain [20] and the Ford-Kac-Mazur model [8], to the sophisticated models of Caldeira and Leggett [5], and Fidaleo and Liverani [6,7]. This class of infinite models is particularly accessible to analysis due to the fact that their phase spaces are symplectic vector spaces, and their dynamics are groups of linear symplectic transformations $[15]$.

For the purpose of quantization the phase space can be made into a complex Hilbert space $\mathcal{H}$, with the imaginary part of the inner product as the symplectic form, and a symplectic group of the form $\mathrm{e}^{\text {ith }}$ for some positive definite 'one particle' Hamiltonian $h$. In a mathematically rigorous form, this approach was pioneered by Segal et al. [13].

The algebra of observables of such an assembly of coupled harmonic oscillators is described by a CCR algebra $\mathcal{A}$ over $\mathcal{H}$, generated by Weyl operators $W(\eta)$, with $\eta \in \mathcal{H}$, satisfying

$$
W\left(\eta_{1}\right) W\left(\eta_{2}\right)=\mathrm{e}^{-\frac{i}{2} \operatorname{Im}\left\langle\eta_{1}, \eta_{2}\right\rangle} W\left(\eta_{1}+\eta_{2}\right),
$$

and with a time evolution $\alpha_{t}$ given by

$$
\alpha_{t}(W(\eta))=W\left(\mathrm{e}^{i t h} \eta\right) .
$$

A KMS state on $\mathcal{A}$ at inverse temperature $\beta$ with respect to this evolution is given by

$$
\omega_{\beta}(W(\eta))=\mathrm{e}^{-\frac{1}{4}\left\langle\eta, \operatorname{coth}\left(\frac{1}{2} \beta h\right) \eta\right\rangle} .
$$

Via the GNS construction this state leads to a representation of $\mathcal{A}$ on a (much larger) Hilbert space $\mathcal{H}_{\beta}$, which possesses a distinguished vector $\xi_{\beta}$ reflecting the KMS state:

$$
\left\langle\xi_{\beta}, A \xi_{\beta}\right\rangle=\omega_{\beta}(A) \quad \text { for } A \in \mathcal{A} .
$$

The relation to approach to equilibrium is the following: If $\mathcal{H}$ is infinitedimensional, which corresponds to infinitely many oscillators, it may happen that for all $\eta_{1}, \eta_{2} \in \mathcal{H}$ :

$$
\lim _{t \rightarrow \infty}\left\langle\eta_{1}, \mathrm{e}^{i t h} \eta_{2}\right\rangle=0 .
$$

As a consequence, the dynamics $\alpha_{t}$ approaches the equilibrium state $\omega_{\beta}$ in the sense of (1). Then the question posed in the introduction arises. We choose a single oscillator, say a vector $q \in \mathcal{H}$, whose position operator $Q$ is given by

$$
W(\lambda q)=\mathrm{e}^{i \lambda Q}
$$


and we perturb the dynamics by adding a term $V:=v(Q)$ to the Hamiltonian on the GNS space. If $v$ is the Fourier transform of some signed measure $\mu$ on $\mathbb{R}$, then

$$
V=v(Q)=\int_{-\infty}^{\infty} \mathrm{e}^{i \lambda Q} \mu(\mathrm{d} \lambda)=\int_{-\infty}^{\infty} W(\lambda q) \mu(\mathrm{d} \lambda)
$$

As discussed in Sect. 1, we are interested in the uniform convergence of the Dyson series for this choice of $V$ and $\alpha_{t}$. In fact it suffices to consider $A \in \mathcal{A}$ of the form $A=W\left(\lambda_{0} \mathrm{e}^{i t_{0} h} q\right)$, for some fixed $t_{0} \in \mathbb{R}$ and $\lambda_{0} \in \mathbb{C}$.

To connect up with the sum of integrals $\Phi$ of Sect. 2, let $f(t):=\mid \operatorname{Im}$ $\left\langle q, \mathrm{e}^{i t h} q\right\rangle \mid$ and $\widetilde{f}(t):=f\left(t-t_{0}\right)$; let the measure $\mu_{+}$be such that $|\mu(S)| \leq \mu_{+}(S)$ for all Borel subsets of $\mathbb{R}$. By repeated use of the equality

$$
\left[W\left(\eta_{1}\right), W\left(\eta_{2}\right)\right]=-2 i \sin \left(\frac{1}{2} \operatorname{Im}\left\langle\eta_{1}, \eta_{2}\right\rangle\right) W\left(\eta_{1}+\eta_{2}\right),
$$

we obtain

$$
\begin{aligned}
& \left\|\left[\alpha_{-t_{n}}(V),\left[\cdots\left[\alpha_{-t_{1}}(V), A\right] \cdots\right]\right]\right\| \\
& \leq \int_{-\infty}^{\infty} \cdots \int_{-\infty}^{\infty}\left|\prod_{j=1}^{n} 2 \sin \frac{1}{2}\left(\sum_{c=0}^{j-1} \operatorname{Im}\left\langle\lambda_{j} \mathrm{e}^{i t_{j} h} q, \lambda_{c} \mathrm{e}^{i t_{c} h} q\right\rangle\right)\right| \mu_{+}\left(\mathrm{d} \lambda_{1}\right) \cdots \mu_{+}\left(\mathrm{d} \lambda_{n}\right) \\
& \leq \sum_{c_{1}=0}^{0} \sum_{c_{2}=0}^{1} \sum_{c_{3}=0}^{2} \cdots \sum_{c_{n}=0}^{n-1}\left(\int_{-\infty}^{\infty} \cdots \int_{-\infty}^{\infty}\left(\prod_{j=1}^{n}\left|\lambda_{j} \lambda_{c_{j}}\right|\right) \mu_{+}\left(\mathrm{d} \lambda_{1}\right) \cdots \mu_{+}\left(\mathrm{d} \lambda_{n}\right)\right) \\
& \quad \times\left(\prod_{j=1}^{n} f_{c_{j}}\left(t_{j}-t_{c_{j}}\right)\right), \\
& =\sum_{c_{1}=0}^{0} \cdots \sum_{c_{n}=0}^{n-1} \widetilde{m}_{\mathrm{d}_{c}(0)} \prod_{j=1}^{n} m_{\mathrm{d}_{c}(j)}\left(\prod_{j=1}^{n} f_{c_{j}}\left(t_{j}-t_{c_{j}}\right)\right)
\end{aligned}
$$

where $d_{c}(j)$ and $f_{i}$ are defined as in (4) and (5) in Sect. 2, and where $\widetilde{m}_{k}:=\left|\lambda_{0}\right|^{k}$ and $m_{k}:=\int_{0}^{\infty}|\lambda|^{k+1} \mu_{+}(\mathrm{d} \lambda)$. Integrating over $t_{1}, t_{2}, \ldots, t_{n}$ and summing over $n$ we obtain the sum $\Phi(\widetilde{m}, m, \widetilde{f}, f)$ in $(3)$.

(It is a pity that, as was shown in [2], this theory still does not allow the perturbation potential $v$ to break the convexity of the oscillator potential, so that metastable oscillator states, which are an important motivation of this type of oscillator models [5], still cannot be treated rigorously.)

\subsection{Interacting Fermionic Open Systems}

As our second example, we shall describe the application of Theorem 1 by Jakšić et al. [11] to prove Green-Kubo formulas and Onsager reciprocity relations for locally interacting fermionic open systems. Their model consists of several Fermi gases at different temperatures and chemical potentials, which interact by a weak 
local interaction potential $V$. Existence and properties of a non-equilibrium stationary state for the total system are proved by showing uniform convergence of the Dyson series. We refer to the paper [11] for further details; let it suffice here to show how in this case the Dyson series is estimated by a sum of integrals of the form $\Phi(\widetilde{m}, m, \widetilde{f}, f)$.

Let $\mathcal{A}$ be the $\mathrm{C}^{*}$-algebra generated by creation and annihilation operators satisfying the canonical anticommutation relations

$$
a\left(\eta_{1}\right) a^{*}\left(\eta_{2}\right)+a^{*}\left(\eta_{2}\right) a\left(\eta_{1}\right)=\left\langle\eta_{1}, \eta_{2}\right\rangle \cdot \mathbf{1},
$$

valid for all $\eta_{1}, \eta_{2}$ in some Hilbert space $\mathcal{H}$. The unperturbed dynamics $\alpha_{t}$ is the automorphism group on $\mathcal{A}$ given by

$$
\alpha_{t}(a(\eta))=a\left(\mathrm{e}^{i t h} \eta\right) .
$$

The perturbation potential is a (self-adjoint) sum over even monomials in annihilation and creation operators

$$
V=\lambda \sum_{k=1}^{K} a^{\#}\left(\vartheta_{k, 1}\right) a^{\#}\left(\vartheta_{k, 2}\right) \cdots a^{\#}\left(\vartheta_{k, q_{k}}\right)
$$

for some positive integer $K$, a positive coupling constant $\lambda$, and even numbers $q_{1}, q_{2}, \ldots, q_{K}$. Here $a^{\#}$ stands for $a$ or $a^{*}$ and the vectors $\vartheta_{k, j}$ are chosen from the unit ball of $\mathcal{H}$. The test observable $A$ is a single monomial of the form $a^{\#}\left(\varphi_{1}\right) \cdots a^{\#}\left(\varphi_{p}\right)$, with $p \in \mathbb{N}$ and $\varphi_{1}, \ldots, \varphi_{m}$ in the unit ball of $\mathcal{H}$.

Now, the commutator of two monomials $A=a_{1} a_{2} \cdots a_{p}$ and $B=b_{1} b_{2} \cdots b_{q}$ of annihilation or creation operators, at least one of which is of even degree, is calculated as

$$
[B, A]=\sum_{i=1}^{p} \sum_{j=1}^{q}(-1)^{j}\left(a_{i} b_{j}+b_{j} a_{i}\right) a_{1} \cdots a_{i-1}\left(b_{1} \cdots b_{j-1} b_{j+1} \cdots b_{q}\right) a_{i+1} \cdots a_{p} .
$$

In particular,

$$
\begin{aligned}
{\left[\alpha_{-t}(V), A\right]=} & \lambda \sum_{k=1}^{K} \sum_{i=1}^{p} \sum_{j=1}^{q_{k}}(-1)^{j}\left\langle\vartheta_{k j}, \mathrm{e}^{i t h} \varphi_{i}\right\rangle_{*} a^{\#}\left(\varphi_{1}\right) \cdots a^{\#}\left(\varphi_{i-1}\right) \\
& \times\left(a^{\#}\left(\mathrm{e}^{-i t h} \vartheta_{k 1}\right) \cdots a^{\#}\left(\mathrm{e}^{-i t h} \vartheta_{k, j-1}\right) a^{\#}\left(\mathrm{e}^{-i t h} \vartheta_{k, j+1}\right) \cdots a^{\#}\left(\mathrm{e}^{-i t h} \vartheta_{k, q_{k}}\right)\right) \\
& \times a^{\#}\left(\varphi_{i+1}\right) \cdots a^{\#}\left(\varphi_{p}\right),
\end{aligned}
$$

where the starred inner product $\left\langle\vartheta_{k j}, \mathrm{e}^{i t h} \varphi_{i}\right\rangle_{*}$ is to be read as 0 when $a^{\#}\left(\varphi_{i}\right)$ and $a^{\#}\left(\vartheta_{k j}\right)$ are both annihilators or both creators, as the ordinary inner product when the first is an annihilator and the second a creator, and as its complex conjugate if it is the other way around. If we now put $\widetilde{f}(t):=\lambda K \max _{i, j, k}\left|\left\langle\vartheta_{k j}, \mathrm{e}^{i t h} \varphi_{i}\right\rangle\right|$, and $q:=\max _{k} q_{k}$, then it follows that

$$
\left\|\left[\alpha_{-t}(V), A\right]\right\| \leq p q \tilde{f}(t) .
$$


Let us also define an upper bound $f(t)$ for the action of $\left[\alpha_{-t}, \cdot\right]$ on $V$ by putting $f(t):=\lambda K \max _{j, k, j^{\prime}, k^{\prime}}\left|\left\langle\vartheta_{k j}, \mathrm{e}^{i t h} \vartheta_{k^{\prime} j^{\prime}}\right\rangle\right|$. Repeated application of (12) then leads to the estimate

$$
\begin{aligned}
& \left\|\left[\alpha_{-t_{n}}(V),\left[\ldots\left[\alpha_{-t_{1}}(V), A\right] \ldots\right]\right]\right\| \\
& \leq \lambda^{n} \sum_{k_{1}=1}^{K} \cdots \sum_{k_{n}=1}^{K} \sum_{c_{1}=0}^{0} \sum_{c_{2}=0}^{1} \sum_{c_{3}=0}^{2} \cdots \sum_{c_{n}=0}^{n-1} p(p-1) \cdots\left(p-d_{c}(0)+1\right) \\
& \quad \times \prod_{j=1}^{n} q_{k_{j}} \cdots\left(q_{k_{j}}-d_{c}(j)\right) \times \prod_{j=1}^{n} f_{c_{j}}\left(t_{j}-t_{c_{j}}\right),
\end{aligned}
$$

where $f_{i}$ is defined as before in (5). Summing over $n \in \mathbb{N}$ and integrating over $t_{1}, t_{2}, \ldots, t_{n}$ with $t_{1} \leq t_{2} \leq \cdots \leq t_{n}$ now yields an estimate of the entire Dyson series by $\Phi(\widetilde{m}, m, \widetilde{f}, f)$, provided we define

$$
\widetilde{m}_{k}:=p(p-1) \cdots(p-k+1) \quad \text { and } \quad m_{k}:=q(q-1) \cdots(q-k) .
$$

The relevant generating functions for this example are

$$
\widetilde{G}(x)=(1+x)^{p} \text { and } G(x)=q(1+x)^{q-1} .
$$

\section{Proof of the Theorem}

\subsection{Rooted Trees}

According to the usual definition a rooted tree is a finite connected graph without cycles and with one distinguished vertex. Here we prefer to use the following, equivalent definition (Fig. 3).

Let $V$ be a finite non-empty set. A rooted tree with vertex set $V$ is a function $a: V \rightarrow V$ with the property that there is a point $\odot \in V$ such that for $k \in \mathbb{N}$ sufficiently large and all $v \in V$ we have $a^{\circ k}(v)=\odot$. The point $\odot$ is called the root of $a$. Note that always $a(\odot)=\odot$. The least value of $k$ for which $a^{\circ k}$ contracts all vertices to the root is the height of the tree $a$. By $V^{*}$ we shall mean $V \backslash\{\odot\}$. In this paper by a tree we always mean a rooted tree.

Drawing an arrow from $v$ to $a(v)$ for each $v \in V^{*}$, we obtain an oriented graph. The number $n$ of arrows will be called the size $|a|$ of the tree. Note that $|a|=\#\left(V^{*}\right)=\#(V)-1$. The vertex set $V$ is partially ordered by $a$ in a natural way: we say that $v \prec w$ if $v=a^{k}(w)$ for some $k \in \mathbb{N}$. We think of $a(v)$ as the parent of $v$ (except, of course, if $v$ is the root). By $d_{a}(v)$ we denote the number of points in $V^{*}$ that are mapped to $v$ by a (the number of children of $v$ if we regard $a$ as a family tree).

Rooted trees $a: V \rightarrow V$ and $b: W \rightarrow W$ are considered isomorphic if there is a bijection $f: V \rightarrow W$ such that $b \circ f=f \circ a$. We denote the collection of all isomorphism classes or types of rooted trees by $\mathbb{A}$. We write $\mathbb{A}_{n}(n \in \mathbb{N})$ for the types of trees of size $n$, and $\mathbb{A}[h](h \in \mathbb{N})$ for the types of trees of height at most $h$. 


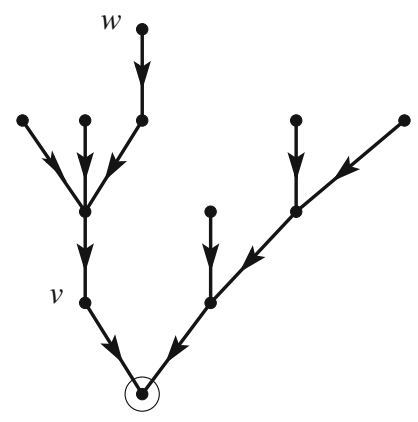

Figure 3. A rooted tree $(v \prec w)$.

We shall denote isomorphism classes of trees by $\alpha, \beta, \ldots$, and write $|\alpha|$ for the size of the trees in class $\alpha$.

An automorphism of a tree $a$ is an isomorphism from $a$ to itself. We denote the group of all automorphisms of $a$ by aut $(a)$. Since all trees of the same type have the same number of automorphisms, we sometimes write \#aut $(\alpha)$ instead of \#aut $(a)$. In the same sense we shall speak of $d_{\alpha}(\odot)$ for a type $\alpha$.

\subsection{Climbers}

The sum (3) contains a summation over functions $c:\{1, \ldots, n\} \rightarrow\{0,1, \ldots, n\}$ : $j \mapsto c(j)=c_{j}$, which are decreasing in the sense that $c(j)<j$ for all $j$. We shall call such maps climbers of size $n$. Note that, if we add the prescription that $c(0)=0$, a climber becomes the same as a rooted tree with vertex set $\{0,1, \ldots, n\}$ satisfying the extra requirement

$$
i \prec j \quad \Longrightarrow \quad i \leq j .
$$

We denote the set of all climbers of size $n$ by $\mathcal{C}_{n}$. We are now going to replace the sum over $\mathcal{C}_{n}$ occurring in (3) by a sum over indexed rooted trees. By an indexation of a rooted tree $a$ on $V$ of size $n$ we mean an order-preserving bijection $\iota: V \rightarrow\{0,1,2, \ldots, n\}$. The set of all indexations of $a$ will be denoted by $I(a)$. Note that for $\iota \in I(a)$ we always have $\iota(\odot)=0$.

By indexation a rooted tree becomes a climber: $c=\iota \circ a \circ \iota^{-1}$. There may be more than one indexation leading to the same climber, as is illustrated in Fig. 4. If $a$ is itself a climber, then $I(a)$ is the set of all isomorphisms of $a$ with isomorphic climbers $c$.

Lemma 4. Let $F: \mathcal{C}_{n} \rightarrow \mathbb{R}$. Then

$$
\sum_{c \in \mathcal{C}_{n}} F(c)=\sum_{\alpha \in \mathbb{A}_{n}} \frac{1}{\# \operatorname{aut}(\alpha)} \sum_{\iota \in I(a)} F\left(\iota \circ a \circ \iota^{-1}\right),
$$

where $a$ is any tree of type $\alpha$. 

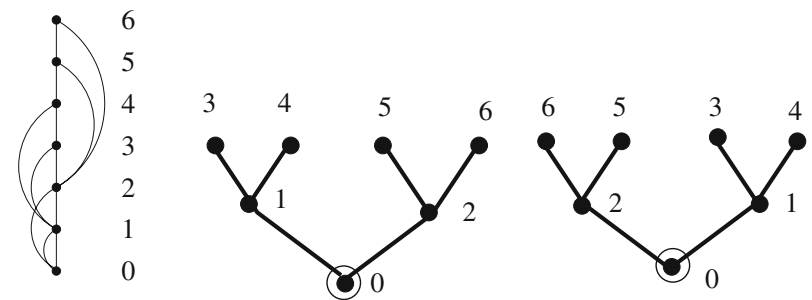

Figure 4. Two indexations of a rooted tree leading to the same climber.

Proof. The set of climbers $\mathcal{C}_{n}$ decomposes into isomorphism classes $\alpha \cap \mathcal{C}_{n}$ with $\alpha \in \mathbb{A}_{n}$. The sum over one such class can be performed by summing over the orbit of a single element $c \in \alpha \cap \mathcal{C}_{n}$ under the group of isomorphisms among climberswhich is equal to the set $I(c)$ of indexations of $c$ as noted above - and then dividing by the number of automorphisms of $c$. Replacing $c \in \alpha \cap \mathcal{C}_{n}$ by an arbitrary tree $a \in \alpha$ we obtain the statement.

Now let

$$
\Delta_{n}:=\left\{t=\left(t_{1}, t_{2}, \ldots, t_{n}\right) \in \mathbb{R}^{n} \mid 0<t_{1}<\cdots<t_{n}\right\} .
$$

The following lemma allows us to replace the summation over $I(a)$ together with the integration over $\Delta_{n}$ by a single integral over $[0, \infty)^{n}$.

Lemma 5 (Packing Lemma). For any rooted tree a with index set $V$ the map

$$
\vartheta_{a}: I(a) \times \Delta_{n} \longrightarrow[0, \infty)^{V^{*}}:(\iota, t) \mapsto r \text {, where } r_{v}:=t_{\iota(v)}-t_{\iota(a(v))},
$$

(with $t_{0}=0$ ) is bijective up to a subset of $[0, \infty)^{V^{*}}$ of measure zero, and has Jacobian 1 on each component $\{\iota\} \times \Delta_{n}$.

Proof. Choose a point $r \in[0, \infty)^{V^{*}}$ and put $r_{\odot}:=0$. Allocate a 'branching time' $s_{v}$ to each vertex $v \in V$ by putting

$$
s_{v}:=\sum_{w \prec v} r_{w} .
$$

If some of these values $s_{v}$ coincide, which happens only for a set of points $r$ of measure 0 , then $r$ is not in the range of $\vartheta_{a}$. If they are all different, they determine by their order a unique indexation $\iota$ of $V$ :

$$
s_{v} \leq s_{w} \quad \Longleftrightarrow \quad \iota(v) \leq \iota(w) .
$$

Putting $t_{\iota(v)}:=s_{v}$ we obtain $t \in \Delta_{n}$ with the property that

$$
\vartheta_{a}(\iota, t)_{v}:=t_{\iota(v)}-t_{\iota(a(v))}=s_{v}-s_{a(v)}=\sum_{w \prec v} r_{w}-\sum_{w \prec a(v)} r_{w}=r_{v} .
$$

So $r$ lies in the range of $\vartheta_{a}$. Conversely, if $r=\vartheta_{a}(\iota, t)$, we must have

$$
t_{\iota(v)}=r_{v}+t_{\iota(a(v))}=r_{v}+r_{a(v)}+t_{\iota(a \circ a(v))}=\cdots=\sum_{w \prec v} r_{w}=s_{v} .
$$




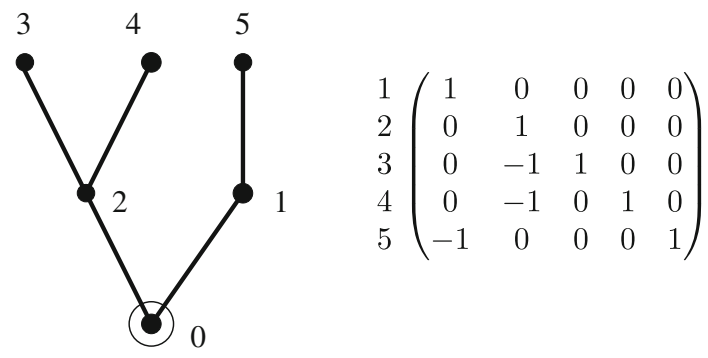

FiguRE 5. A indexed rooted tree $(a, \iota)$ with its matrix $\left(M_{v, k}\right)$.

And since $0<t_{1}<t_{2}<\cdots<t_{n}$, the indexation $\iota$ is uniquely determined by the order of the 'branching times' $s_{v}$, hence by $r$. So $\vartheta_{a}$ is injective as well as almost surjective.

Finally, the map $t \mapsto \vartheta_{a}(\iota, t)$, for $\iota \in I(a)$ fixed, can be written as a $V^{*} \times n$ matrix $\left(M_{v, k}\right)$, which has 1's at the positions $(v, k)$, where $\iota(v)=k,(-1)$ 's at the positions $(v, k)$ with $\iota(a(v))=k$ and 0 's everywhere else. We may put $M$ in standard form by ordering the points in $V^{*}$ according to the indexation $\iota$, thus putting all the 1's on the diagonal (cf. Fig. 5).

Then since $\iota(a(v))<\iota(v)$, all the $(-1)$ 's end up below the diagonal. So $\operatorname{det}(M)$ equals 1 in this standard form, and \pm 1 in any other ordering of $V^{*}$. The Jacobian $|\operatorname{det}(M)|$ of the piecewise linear map $\vartheta_{a}$ equals 1 almost everywhere.

Lemma 6. The sum of integrals $\Phi(\widetilde{m}, m, \tilde{f}, f)$ in $(3)$ can be written as

$$
\Phi(\widetilde{m}, m, \tilde{f}, f)=\sum_{\alpha \in \mathbb{A}} \frac{\tilde{m}_{d_{\alpha}(\odot)}\|\widetilde{f}\|^{d_{\alpha}(\odot)}}{\# \operatorname{aut}(\alpha)}\left(\prod_{v \in V^{*}} m_{d_{a}(v)}\|f\|^{d_{a}(v)}\right),
$$

where the tree a with vertex set $V$ is any representative of the class $\alpha$.

Proof. In (3) $\Phi(\widetilde{m}, m, \tilde{f}, f)$ is written as a sum over $n$ of sums of integrals over $\mathcal{C}_{n} \times \Delta_{n}$. First we apply Lemma 4 to replace the sum over $\mathcal{C}_{n}$ by a sum over indexed rooted trees, to obtain

$$
\begin{aligned}
\Phi(\widetilde{m}, m, \tilde{f}, f)= & \sum_{n=0}^{\infty} \sum_{\alpha \in \mathbb{A}_{n}} \frac{\widetilde{m}_{d_{\alpha}(\odot)}}{|\operatorname{aut}(\alpha)|}\left(\prod_{v \in V^{*}} m_{d_{a}(v)}\right) \\
& \times \sum_{\iota \in I(a)} \int_{\Delta_{n}}\left(\prod_{v \in V^{*}} f_{\iota(a(v))}\left(t_{\iota(v)}-t_{\iota(a(v))}\right)\right) \mathrm{d} t,
\end{aligned}
$$

where again $a: V \rightarrow V$ is any tree of type $\alpha$. Then we apply the 'packing lemma' (Lemma 5) to replace the sum over $\iota$ and the integration over $t$ by an integration over $r$ : 


$$
\Phi(\widetilde{m}, m, \tilde{f}, f)=\sum_{\alpha \in \mathbb{A}} \frac{\widetilde{m}_{d_{\alpha}(\odot)}}{|\operatorname{aut}(\alpha)|}\left(\prod_{v \in V^{*}} m_{d_{a}(v)}\right) \int_{[0, \infty)^{*}}\left(\prod_{v \in V^{*}} f_{a(v)}\left(r_{v}\right)\right) \mathrm{d} r
$$

where $a: V \rightarrow V$ is any tree of type $\alpha$, and for $x \in V: f_{x}:=\tilde{f}$ if $x$ is the root, otherwise $f_{x}:=f$. The integral over $r$ is now easily obtained, and the Lemma is proved.

Now for $\widetilde{m}, m, \widetilde{f}$ and $f$ fixed, let the weight $\widetilde{w}(a)$ of a rooted tree $a$, be as it occurs in Lemma 6:

$$
\widetilde{w}(a):=\widetilde{m}_{d_{a}(\odot)}\|\widetilde{f}\|^{d_{a}(\odot)} \prod_{v \in V^{*}} m_{d_{a}(v)}\|f\|^{d_{a}(v)} .
$$

By $w(a)$ we shall denote the same weight, but with $\widetilde{f}=f$ and $\widetilde{m}=m$, which simplifies to

$$
w(a)=\prod_{v \in V} m_{d_{a}(v)}\|f\|^{d_{a}(v)} .
$$

Both $w(a)$ and $\widetilde{w}(a)$ depend only on the type of $a$, hence we may write $w(\alpha)$ and $\widetilde{w}(\alpha)$, respectively.

Let $\mathbb{A}[h]$ with $h \in \mathbb{N}$ denote the set of all rooted trees of height $\leq h$, and define

$$
\Phi_{h}(\widetilde{m}, m, \tilde{f}, f):=\sum_{\alpha \in \mathbb{A}[h]} \frac{\widetilde{w}(\alpha)}{|\operatorname{aut}(\alpha)|} .
$$

Lemma 7. For all pairs of sequences $\widetilde{m}, m$ of non-negative numbers, all pairs of integrable functions $f, \widetilde{f}:[0, \infty) \rightarrow[0, \infty)$ and all $h \in \mathbb{N}$,

$$
\Phi_{h+1}(\widetilde{m}, m, \widetilde{f}, f)=\widetilde{G}\left(\|\widetilde{f}\| \Phi_{h}(m, m, f, f)\right),
$$

where $G$ and $\widetilde{G}$ are the generating functions given in (6).

Proof. If $a$ is a rooted tree of height at most $h+1$ and root degree $d_{a}(\odot)=p$, and we cut off its root, then we are left with a set of $p$ rooted trees of height at most $h$. On the level of isomorphism classes this leads to a one-to-one correspondence between $\mathbb{A}[h+1]$ and $p$-multisets from $\mathbb{A}[h]$, i.e. functions $\mu: \mathbb{A}[h] \rightarrow \mathbb{N}$ which satisfy

$$
\sum_{\beta \in \mathbb{A}[h]} \mu(\beta)=p
$$

Under this correspondence we have

$$
\widetilde{w}(\alpha)=\widetilde{m}_{p}\|\widetilde{f}\|^{p} \cdot \prod_{\beta \in \mathbb{A}[h]} w(\beta)^{\mu(\beta)},
$$

and

$$
\# \operatorname{aut}(\alpha)=\prod_{\beta \in \mathbb{A}[h]} \mu(\beta) !(\# \operatorname{aut}(\beta))^{\mu(\beta)}
$$


since any automorphism of a tree of type $\alpha$ amounts to a permutation of isomorphic subtrees, plus the application of an automorphism to each of them. We calculate, starting from Lemma 6, and applying the multinomial formula to $\mu$,

$$
\begin{aligned}
\Phi_{h+1}(\tilde{m}, m, \tilde{f}, f) & =\sum_{\alpha \in \mathbb{A}[h+1]} \frac{\widetilde{w}(\alpha)}{\# \operatorname{aut}(\alpha)} \\
& =\sum_{p=0}^{\infty} \widetilde{m}_{p}\|\widetilde{f}\|^{p} \sum_{\substack{\mu: \mathbb{A}[h] \rightarrow \mathbb{N} \\
\sum \mu=p}} \prod_{\beta \in \mathbb{A}[h]} \frac{w(\beta)^{\mu(\beta)}}{\mu(\beta) !(\# \operatorname{aut}(\beta))^{\mu(\beta)}} \\
& =\sum_{p=0}^{\infty} \tilde{m}_{p}\|\widetilde{f}\|^{p} \frac{1}{p !}\left(\sum_{\beta \in \mathbb{A}[h]} \frac{w(\beta)}{\# \operatorname{aut}(\beta)}\right)^{p} \\
& =\widetilde{G}\left(\|\widetilde{f}\| \Phi_{h}(m, m, f, f)\right) .
\end{aligned}
$$

Proof of Theorem 1. Let $G_{f}$ and $\widetilde{G}_{\tilde{f}}$ denote the maps

$$
y \mapsto G(\|f\| y) \quad \text { and } \quad y \mapsto \widetilde{G}(\|\widetilde{f}\| y) .
$$

By Lemma 7, and since $\Phi_{0}(m, m, f, f)=m_{0}=G_{f}(0)$, we have for all $h \in \mathbb{N}$ :

$$
\Phi_{h}(\widetilde{m}, m, \tilde{f}, f)=\widetilde{G}_{\tilde{f}} \circ G_{f}^{\circ h}(0) .
$$

We must study convergence of this expression as $h \rightarrow \infty$. First consider $y_{h}:=$ $G_{f}^{\circ h}(0)$. Suppose that $(7)$ has a solution, i.e. $G_{f}$ has a fixed point $u \geq 0$. Then, since $G_{f}$ is non-decreasing, and since $0 \leq u$, we have for all $h \geq 1$ :

$$
y_{h}=G_{f}^{o h}(0) \leq G_{f}^{\circ h}(u)=u .
$$

Being bounded above, the sequence converges to a limit $y \leq u$. As $y$ must be a fixed point itself, it is the least such point.

On the other hand, if (7) has no solution, the sequence $y_{1}, y_{2}, y_{3}, \ldots$ can have no finite limit, so it must tend to infinity.

Finally, consider the sequence $\left(\widetilde{G}_{\tilde{f}}\left(y_{h}\right)\right)_{h \in \mathbb{N}}$ in $(13)$. By assumption, $\widetilde{m}_{1}, \widetilde{m}_{2}, \ldots$ are not all zero, hence $\widetilde{G}_{\tilde{f}}$ is strictly increasing and convex. This implies that the limit

$$
\Phi(\widetilde{m}, m, \tilde{f}, f)=\lim _{h \rightarrow \infty} \Phi_{h}(\widetilde{m}, m, \tilde{f}, f)=\lim _{h \rightarrow \infty} \widetilde{G}_{\tilde{f}}\left(y_{h}\right)
$$

exists iff the increasing sequence $y_{h}$ converges to a point $y$ in the region of convergence of $\widetilde{G}_{\tilde{f}}$. As argued above, $y$ is the least fixed point of $G_{f}$ if such exists. We conclude that the sequence (13) converges if and only if the least fixed point $y$ of $G_{f}$ lies in the domain of $\widetilde{G}_{\tilde{f}}$; in that case,

$$
\Phi(\widetilde{m}, m, \tilde{f}, f)=\widetilde{G}_{\tilde{f}}(y) .
$$




\section{Acknowledgement}

We thank the anonymous referee for corrections and valuable suggestions for improvement.

Open Access. This article is distributed under the terms of the Creative Commons Attribution Noncommercial License which permits any noncommercial use, distribution, and reproduction in any medium, provided the original author(s) and source are credited.

\section{References}

[1] Botvich, D., Fayolle, G., Malyshev, V.: Loss networks in thermodinamic limit. In: 11th Conference of Analysis and Optimisation of Systems: Discrete Event Systems. Sopia-Antipolis France 1994. Lecture Notes in Control and Information Science, vol. 199, pp. 465-489. Springer, Berlin (1994)

[2] Botvich, D., Guţă, M., Maassen, H.: Stability of Bose Dynamical Systems and Branching Theory. Preprint (mp-arc 99-130)

[3] Botvich, D., Malyshev, V.A.: Unitary equivalence of temperature dynamics for ideal and locally perturbed Fermi gas. Commun. Math. Phys. 91, 301-312 (1983)

[4] Bergeron, F., Labelle, G., Leroux, P.: Combinatorial Species and Tree-Like Structures. Cambridge University Press, Cambridge (1998)

[5] Caldeira, A.C., Leggett, A.J.: Influence of dissipation on quantum tunneling in microscopic systems. Phys. Rev. Lett. 46, 211-214 (1981)

[6] Fidaleo, F., Liverani, C.: Ergodic properties for a quantum non linear dynamics. J. Stat. Phys. 97, 957-1009 (1999)

[7] Fidaleo, F., Liverani, C.: Ergodic properties of a model related to disordered quantum anharmonic crystals. Commun. Math. Phys. 235, 169-189 (2003)

[8] Ford, G., Kac, M., Mazur, P.: Statistical mechanics of assemblies of coupled oscillators. J. Math. Phys. 6, 504-515 (1965)

[9] Fröhlich, J., Merkli, M., Ueltchi, D.: Dissipative transport: thermal contacts and tunneling junctions. Ann. Henri Poincaré 4, 897-945 (2004)

[10] Harris, T.E.: The Theory of Branching Processes. Springer, Berlin (1963)

[11] Jakšić, V., Ogata, Y., Pillet, C.-A.: The Green-Kubo formula for locally interacting fermionic open systems. Ann. Henri Poincaré 8, 1013-1036 (2007)

[12] Joyal, A.: Une théorie combinatoire des series formelles. Adv. Math. 42, 1-82 (1981)

[13] Kay, B.S.: A uniqueness result in the Segal-Weinless approach to linear Bose fields. J. Math. Phys. 20, 1712-1713 (1979)

[14] Lamb, H.: On a peculiarity of the wave-system due to the free vibrations of a nucleus in an extended medium. Proc. Lond. Math. Soc. 2, 88 (1900)

[15] Lewis, J.T., Maassen, H.: Hamiltonian models of classical and quantum stochastic processes. In: Quantum Probability and Applications to the Quantum Theory of Irreversible Processes. Lecture Notes in Mathematics, vol. 1055. Springer, Berlin (1984) 
[16] Maassen, H.: On the invertibility of Møller morphisms. J. Math. Phys. 23, 1848-1851 (1982)

[17] Maassen, H.: Return to equilibrium by a solution of a quantum Langevin equation. J. Stat. Phys. 34, 239-262 (1984)

[18] Robinson, D.W.: Return to equilibrium. Commun. Math. Phys. 31, 171-189 (1973)

[19] Spohn, H.: Asymptotic completeness for Rayleigh scattering. J. Math. Phys. 38, 2281-2296 (1997)

[20] van Hemmen, J.L.: Dynamics and Ergodicity of the Infinite Harmonic Crystal, a Review of some Salient Features. Springer Lecture Notes in Physics, vol. 93. Springer, Berlin (1979)

Hans Maassen

Department of Mathematics

Radboud University Nijmegen

Heyendaalseweg 135

6525 AJ Nijmegen

The Netherlands

e-mail: maassen@math.ru.nl

Dmitri Botvich

Telecommunications Software and Systems Group

Waterford Institute of Technology

Carriganore Campus

Waterford

Ireland

e-mail: dbotvich@tssg.org

Communicated by Claude Alain Pillet.

Received: October 30, 2007.

Accepted: September 15, 2009. 